\title{
Assessing the effect of shape on the evaluation of expected and actual chocolate flavour
}

\author{
Qian Janice Wang ${ }^{1 *}$, Felipe Reinoso Carvalho ${ }^{2,3}$, Dominique Persoone ${ }^{4}$ and Charles Spence ${ }^{1}$
}

\begin{abstract}
Background: Shape can play an important role in our perception of food. In this study, the consequences of crossmodal correspondences between shape and taste on the expected and actual experience of eating chocolate were evaluated. Participants were given two identical dark chocolates differing only in terms of their shape (round or angular) and then rated their expectations of sweetness, bitterness, creaminess and liking. Subsequently, they rated their experience of those attributes on tasting the chocolates. Repeated-measures multivariate analyses (RM-MANOVA) of variance were conducted to assess the role of chocolate shape and cacao content on expected and post-taste chocolate ratings. In addition, we assessed the differences between expected and post-taste ratings for each chocolate shape.

Results: The results revealed that the shape of the chocolate significantly influenced expected sweetness, bitterness and creaminess. By contrast, it had no significant effect on post-tasting ratings. The round chocolates were judged to be less sweet, more bitter, less creamy and liked less than expected, whereas the angular chocolates were judged to be slightly more creamy than expected.

Conclusions: The theory of assimilation and contrast is outlined as one plausible mechanism behind the differences between expectation and the actual taste experience. Implications for food product design and directions for future research are discussed.
\end{abstract}

Keywords: Crossmodal correspondences, Expectations, Taste, Shape, Mouthfeel, Chocolate

\section{Background}

Shape can play an important role in our perception of food. Many studies have shown that people can reliably match basic tastes to shapes with varying degrees of angularity (see [1], for a review). Most prominent amongst these correspondences is the association between round shapes and sweetness [2-4]. Round shapes have even been shown to increase participants' sensitivity for sweetness at threshold levels, compared to angular shapes $[5,6]$. In the realm of chocolate, people from Western cultures tend to associate chocolate with a higher cacao content (e.g. dark chocolate, which tend to be more bitter) with more angular shapes and chocolate with lower cacao content (e.g. milk chocolate, which

\footnotetext{
* Correspondence: qian.wang@psy.ox.ac.uk

${ }^{1}$ Crossmodal Research Laboratory, Department of Experimental Psychology,

University of Oxford, South Parks Road, Oxford OX1 3UD, UK

Full list of author information is available at the end of the article
}

tend to be sweeter) with rounder shapes [7-9]; interestingly, the remote Himba tribe in Northern Namibia makes the opposite mapping, with milk chocolate mapped to more angular shapes [10]. Food perception can even be influenced by the shape of the plate it is served on. For instance, different foods have been shown to taste sweeter when sampled from a round plate than from a square one $([11,12]$; see [13], for a review; though see [14] for a null effect of plate shape on taste ratings).

Furthermore, such shape-taste associations can influence consumer expectations of food products. For instance, round packaging has been shown to give rise to higher expected sweetness than packaging that is more angular (e.g. [15]; see [16], for a recent review). Speaking more generally, round packaging tends to be preferred over angular packaging [17]. People also expect a sweeter-tasting product on viewing a rounder design 
and more sour tastes when exposed to a more angular design ([18]; see also [16, 19] for reviews). The ability for shape to influence expectations may be explained via affective priming. Round shapes are perceived as more pleasant than angular shapes [20, 21]. Furthermore, there is electrophysiological evidence that geometric shapes may automatically activate an emotional response [22].

Finally, such sensory expectations have been shown to alter the sensory perception and hedonic evaluation of the actual food product (see [23, 24] for reviews). Many theories have been put forward to account for the effect of disparity between expectations and the subsequent eating experience. In particular, the theory of assimilation contrast has been used by food science researchers [25]. According to the theory, if the difference between expectation and reality is within the consumer's limit of acceptance, the consumer would, consciously or otherwise, try to change their perception of the product to bring it in line with expectations. On the other hand, if the difference is sufficiently great, the consumer exaggerates the difference between expectations and reality, and their product evaluation shifts in the opposite direction than originally expected [26, 27]. For instance, a slight understatement of quality in advertising might lead to higher consumer satisfaction with the product, and an overstated promise of quality for a poor product might lead to greater customer disappointment.

With all this in mind, it certainly seems plausible that a change in shape might influence the way in which people perceive and experience food. We chose to focus on chocolate since it is a popular food item that is sold in a variety of shapes. In the present study, therefore, we set out to test the hypothesis that shape might alter both the expected and actual sensory and hedonic experience of eating chocolate.

\section{Results}

\section{Expectations}

The RM-MANOVA test revealed a significant main effect of shape (see Fig. 1) on participants' ratings of the chocolates' expected flavour attributes and preference $(F(4,97)=14.64, p<0.001$, Wilks' lambda $=0.62)$, but no effect of cacao content $(F(4,97)=1.52, p=0.20)$. Further ANOVAs revealed significant effects of chocolate shape on measures of sweetness $(F(1,100)=33.62, p<0.001$, $\left.\eta_{\text {partial }}^{2}=0.25\right), \quad$ bitterness $(F(1,100)=23.41, \quad p \leq 0.001$, $\left.\eta_{\text {partial }}^{2}=0.19\right)$ and creaminess $(F(1,100)=50.56, p<0.001$, $\left.\eta_{\text {partial }}^{2}=0.34\right)$, but not for chocolate liking $(F(1,100)=0.03$, $p=0.87)$. In general, round shapes were rated as sweeter, less bitter and creamier than the angularshaped chocolates.

\section{Post-tasting ratings}

The RM-MANOVA test revealed a significant main effect of cacao content (71 or $80 \%$ ) on participants' chocolate ratings $(F(4,97)=3.51, \quad p=0.01$, Wilks' lambda $=0.87$ ), but no effect of shape was found $(F(4,97)=1.57, p=0.19)$. Further ANOVAs revealed significant effects of cacao content on bitterness ratings $\left(F(1,100)=7.50, p=0.007, \quad \eta_{\text {partial }}^{2}=0.07\right)$, where the $71 \%$ chocolate was rated as more bitter than the $80 \%$ chocolate. There were no significant effects of cacao content on sweetness $(F(1,100)=2.58, p=0.11)$, creaminess $(F(1,100)=0.65, p=0.42)$ or chocolate liking $(F(1,100)=0.10, p=0.76)$.

\section{Expected vs. post-tasting ratings}

The RM-MANOVA test revealed significant main effects of rating type $(F(4,98)=6.61, p<0.001$, Wilks' lambda $=0.79)$. Further ANOVAs revealed significant effects of rating type (expected or actual) on measures of sweetness $\left(F(1,101)=21.27, p<0.001, \eta_{\text {partial }}^{2}=0.17\right)$, bitterness $\left(F(1,101)=6.37, p=0.01, \eta_{\text {partial }}^{2}=0.06\right)$ and liking $\left(F(1,101)=9.39, \quad p=0.003, \quad \eta_{\text {partial }}^{2}=0.09\right)$, but not for creaminess $(F(1,101)=1.50, p=0.22)$.

More specifically, the RM-MANOVA test also revealed a significant interaction effect between rating type and shape $(F(4,98)=7.73, p<0.001$, Wilks' lambda $=0.76)$ (see Fig. 2), with regard to sweetness $(F(1,101)=19.71$, $\left.p<0.001, \quad \eta_{\text {partial }}^{2}=0.16\right)$, bitterness $(F(1,101)=12.24$, $\left.p=0.001, \eta_{\text {partial }}^{2}=0.11\right)$ and creaminess $(F(1,101)=22.27$, $\left.p<0.001, \eta_{\text {partial }}^{2}=0.18\right)$. Further post hoc testing showed that the round chocolates were less sweet, more bitter, less creamy and liked less than expected ( $p<0.01$ for all comparisons); on the other hand, angular chocolates were more creamy than expected $(p=0.05)$. These ratings are shown in Fig. 2.

\section{Discussion}

The results of the present study demonstrate that, compared to angular-shaped chocolate, participants expected the rounder chocolate to be significantly sweeter, less bitter and creamier. However, when it comes to posttaste chocolate ratings, there were no significant differences between shapes in the present study. Furthermore, the round chocolates were significantly less sweet, more bitter, less creamy and liked less than expected, while the post-taste ratings for angular chocolates were more creamy than expected, but otherwise not significantly different from expectations. This discrepancy between expectations and reality could have induced a contrast effect, whereby the participants would have further magnified the difference between expectations and reality (see $[26,28])$. The fact that the round chocolate was liked less than expected is also in line with the contrast hypothesis, according to which the disconfirmation of 


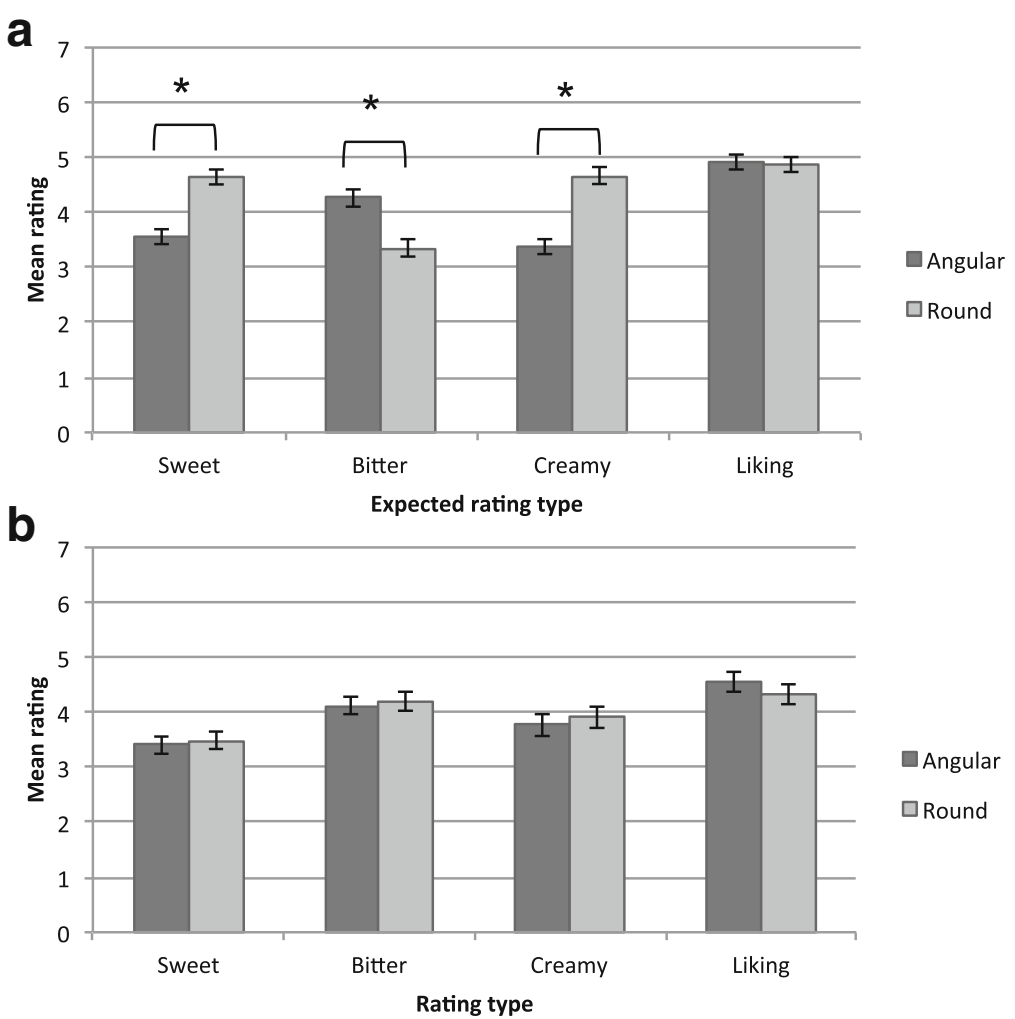

Fig. 1 Two shapes of chocolates used during the study. The round shape was hemispherical while the angular shape was a seven-sided prism. Note that the 71 and $80 \%$ cacao chocolates appear to have the same colour

expectations would lead to a decreased hedonic evaluation [29]. Overall, these results are consistent with the literature showing that round shapes can prime specific taste/flavour expectations, which could then go on to influence the subsequent tasting experience [15].

While the rounder-shaped chocolates did indeed prime expectations that chocolate will taste sweeter and creamier, those expectations did not carry over to influence the actual tasting experience. One possible explanation is that the chocolate samples used in the present study were simply too dark and differed too much from participants' expectations. As a case in point, the mean expected sweetness of the round chocolates was 4.64/7, whereas the actual sweet rating was only 3.48/7. As we have seen in previous studies, expectations have a greater influence on sensory perception when the discrepancy between expectations and reality is not too large [30,31]. In future research, it would be worth repeating the present study with exactly the same shapes, but using a sweeter milk chocolate, in order to test for an assimilation effect of the enhanced sweetness and creaminess ratings.

The fact that the $71 \%$ chocolate was rated to be more bitter than the $80 \%$ chocolate might initially seem surprising, but the effect is small [32], and we did not control for supertaster status between the two groups of participants who tasted 71 vs. $80 \%$ see (Fast $\mathrm{K}$ : Developing a scale to measure just about anything: comparisons across groups and individuals, unpublished M.D. thesis, for evidence of differing chocolate bitterness ratings as a function of taster status, and [33], on the general problem of between-group comparisons of perceived taste intensity given differences in taster status). Another plausible explanation is that participants might have found the $71 \%$ chocolate to taste more acidic and confounded acidity in the chocolates with bitterness (e.g., [34]). Furthermore, it is plausible that the crystalline structure ${ }^{1}$, and therefore the mouthfeel, of the chocolates could have varied across samples. Although, as the chocolates were produced in the workshop of a master chocolatier, it is likely that chocolate texture was consistent across all the samples.

Another potentially confounding factor in the present study is whether the expectation values were driven by angularity or asymmetry, since the angular chocolate used here also happened to be asymmetrical. It has been shown that, along with angular shapes, asymmetrical shapes also tend to be associated with unpleasantness and sourness (as opposed to pleasantness and sweetness) by participants from both the UK and Colombia [35]. 


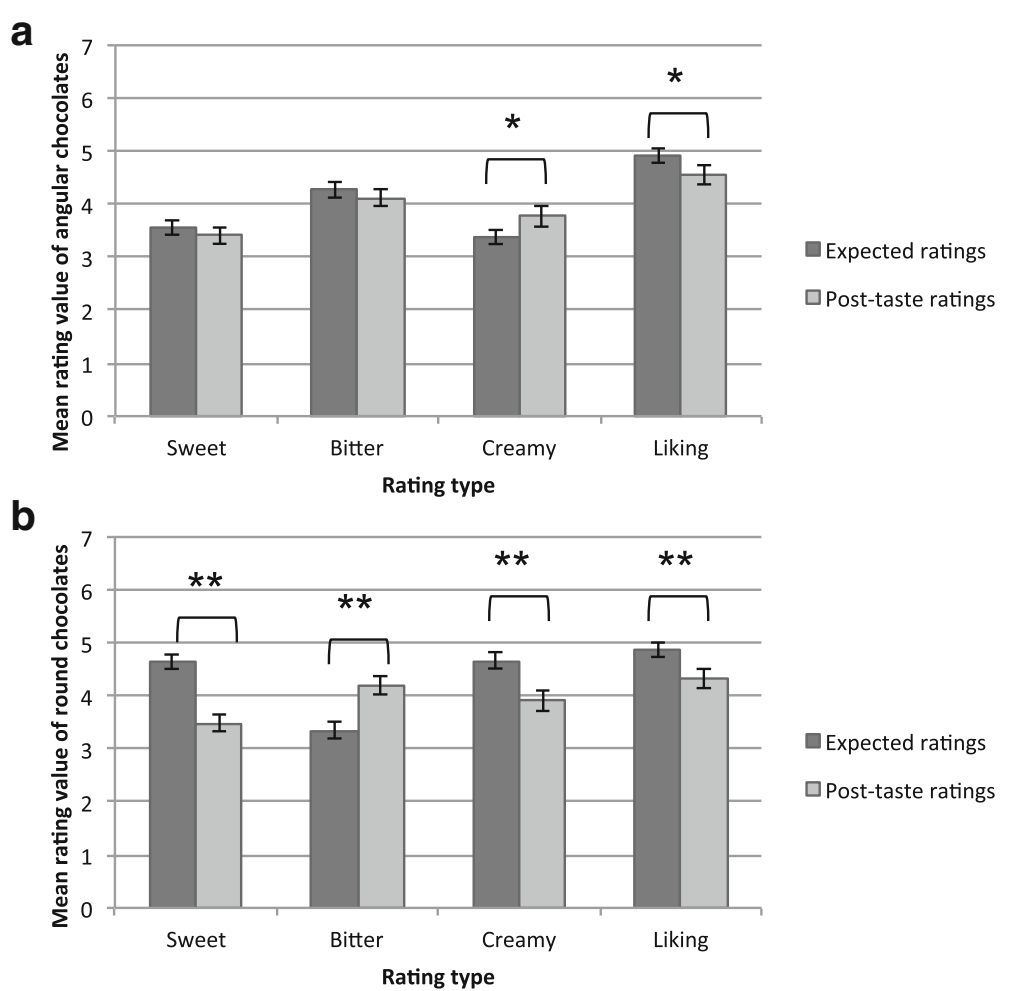

Fig. 2 Mean values of $\mathbf{a}$ expected and $\mathbf{b}$ actual ratings of sweetness, bitterness, creaminess and liking, for both angular and round chocolate shapes. Error bars indicate the standard error. Asterisk ${ }^{{ }^{* \prime \prime}}$ indicates statistical significance at $p<0.05$

Therefore, it would be interesting in future studies to control for symmetry when selecting chocolate shapes.

\section{Conclusions}

In summary, the present study demonstrates the importance of product shape on setting consumer expectations. Chocolates with round shapes were expected to be sweeter, less bitter and more creamy than angularshaped chocolates. In the age of constant product upgrades, it is increasingly important for food manufacturers and designers to take such considerations into account. For instance, Cadbury has recently updated two of its products to have smoother contours, with the claim that the new smoother contours will be a better fit for the mouth [36]. In addition, Cadbury claims the new shapes allow the chocolates to melt in the mouth slightly before biting, for maximum flavour and prolonged enjoyment (see [37] for evidence of chocolate shape's influence on texture and flavour perceptions). It remains to be seen how this new product updating will affect the overall consumer's experience (see [38]).

\section{Methods}

\section{Participants}

One hundred two participants (58 women, 44 men) aged between 16 and 74 years $(M=35.64, \mathrm{SD}=16.81)$ took part in the study. All of the participants gave their informed consent prior to taking part. The participants did not have a cold nor any other known impairment of their sense of smell, taste or hearing at the time of the study, by self-report. The participants were informed that they would be tasting chocolate before the start of the study.

\section{Food stimuli}

Two bitter chocolate formulas were prepared for use in this study (basic ingredients: cocoa mass, sugar, cocoa butter and natural vanilla flavour). One formula had 71\% cocoa and the other $80 \%$. Furthermore, each formula was presented in two different shapes, one rounded and the other angular (see Fig. 3). The chocolates were developed at The Chocolate Line factory in Bruges, under the supervision of the award-wining Belgian chocolatier Dominique Persoone (www.thechocolateline.be). Note that all of the chocolate samples had the same dark brown colour (although due to its geometry, the round shape looks more shiny) and similar volume (approximately $2.5 \mathrm{~cm}^{3}$ ).

\section{Design}

The study was approved by the Social Ethics Committee at KU Leuven-SMEC (Protocol G-2016 03 519). 



Round

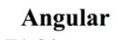

$71 \%$ cocoa
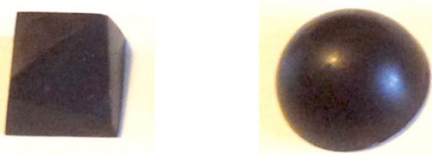

Angular

$80 \%$ cocoa
Round

$80 \%$ cocoa

Fig. 3 Mean values of pre-taste (expected) and post-taste ratings of sweetness, bitterness, creaminess and liking, for a angular and $\mathbf{b}$ round chocolate shapes. Error bars indicate the standard error. Single asterisk ${ }^{\prime * \prime}$ indicates statistical significance at $p \leq .05$ and double asterisks ${ }^{* *}$ indicate statistical significance at $p<.005$

Participants were given two pieces of chocolate with different shapes (but with identical formulas) and rated, first, the expected sweetness, bitterness, creaminess and liking for both pieces of chocolate. After tasting, they repeated the same ratings for both chocolates. Half of the participants tasted the $71 \%$ cacao formula and the rest tasted the $80 \%$ cacao formula.

\section{Procedure}

The ninth floor of Music Instrument Museum in Brussels (MIM) was chosen as the site for the study. Due to its independent location inside the museum, being located between the museum's restaurant on the top floor and the rest of the exhibitions below, it was possible to maintain a reasonably well-controlled environment during the study.

Each participant was seated in front of a computer screen and given two chocolates on labelled plates (1 and 2), tap water, a computer mouse and a keyboard to interact with the survey. The participants were randomly assigned to taste either the $71 \%$ or the $80 \%$ chocolates. First, the participants were instructed to look at (but not taste) the first piece of chocolate and evaluate its expected sweetness, bitterness, creaminess and liking, on 7 -point Likert scales. They then moved on to the second piece of chocolate and repeated the procedure. Next, the participants were instructed to go back to the first piece of chocolate, taste it and then rate its sweetness, bitterness, creaminess and how much they liked it. After rinsing their mouths out with water to cleanse their palates, the participants then tasted and rated the second piece of chocolate. The order in which the chocolates were tasted was randomised amongst participants.

Together with the written guidelines concerning the study, at least one supervisor was present during the study in order to provide guidance and support. Upon finishing the study, the participants were then instructed to leave the room without discussing any details with the next group of participants. The study lasted for around $10 \mathrm{~min}$.

\section{Data analysis}

For both expected and post-tasting chocolate ratings, a repeated-measures multivariate analysis of variance (RM-MANOVA) was conducted on the measures of sweetness, bitterness, creaminess and liking. Shape (round or angular) was the within-participant factor, and cacao content (71 or $80 \%$ ) was the between-participant factor. Expected chocolate ratings were also compared to the post-tasting ratings via a RM-MANOVA, with rating type (before or after tasting) and chocolate shape as within-participant factors and sweetness, bitterness, creaminess and liking as dependent variables. All post hoc pairwise comparisons are Bonferroni corrected.

\section{Endnote}

${ }^{1}$ Solid chocolate has a crystalline structure formed by cocoa butter. Tempering is a precise, temperaturesensitive process used to bring cocoa butter into a stable crystalline form [39]. Most manufactured chocolates have been tempered to give them hardness and shine.

\section{Acknowledgements}

The authors would like to thank François Nelissen, Jo Santy and Maite

Bezunartea for their contribution during data collection. We would also like to thank the crew from The Chocolate Line, for producing and donating the chocolate samples used in this study and the MIM crew for their kind support during the development of the study at the museum.

\section{Funding}

CS would like to thank the AHRC grant entitled 'Rethinking the senses' (AH/L007053/1) for supporting this research. FRC was partly funded by the CAPES Foundation, Brazil (BEX 3488/13-6).

Availability of data and materials

The data supporting the conclusions of this article will be available in the Oxford University Research Archive (https://ora.ox.ac.uk/).

\section{Authors' contributions}

QJW and FRC designed the study. FRC collected the data. DP designed the experimental stimuli. QJW and FRC performed data analysis. QJW, FRC and CS participated in the manuscript preparation. All authors read and approved the final manuscript.

\section{Competing interests}

The authors declare that they have no competing interests.

Consent for publication

The authors consent for the publication of this article in Flavour. 


\section{Ethics approval and consent to participate}

Ethics approval was obtained for this study through the Social Ethics Committee at KU Leuven-SMEC (Protocol G-2016 03 519). Participants consented to participation.

\section{Author details}

'Crossmodal Research Laboratory, Department of Experimental Psychology, University of Oxford, South Parks Road, Oxford OX1 3UD, UK. ${ }^{2}$ Department of Electronics and Informatics (ETRO), Vrije Universiteit Brussel, Brussels, Belgium. ${ }^{3}$ Department of Experimental Psychology, KU Leuven, Leuven, Belgium. ${ }^{4}$ The Chocolate Line, Bruges, Belgium.

Received: 18 September 2016 Accepted: 6 December 2016 Published online: 08 February 2017

\section{References}

1. Spence C, Ngo MK. Assessing the shape symbolism of the taste, flavour, and texture of foods and beverages. Flavour. 2012;1:12.

2. Ngo MK, Velasco C, Salgado A, Boehm E, O'Neill D, Spence C. Assessing crossmodal correspondences in exotic fruit juices: the case of shape and sound symbolism. Food Qual Prefer. 2013;28:361-9.

3. Obrist, M., Comber, R., Subramanian, S., Piqueras-Fiszman, B., Velasco, C., \& Spence, C. (2014). Temporal, affective, and embodied characteristics of taste experiences. In: Proceedings of the 32nd Annual ACM Conference on Human Factors in Computing Systems, 2853-2862.

4. Velasco C, Woods A, Deroy O, Spence C. Hedonic mediation of the crossmodal correspondence between taste and shape. Food Qual Prefer. 2014;41:151-8.

5. Liang P, Roy S, Chen M-L, Zhang G-H. Visual influence of shapes and semantic familiarity on human sweet sensitivity. Behav Brain Res. 2013;253:42-7.

6. Liang, P., Biswas, P., Vinnakota, S. et al. (2016). Invariant effect of vision on taste across two Asian cultures: India and China. J Sens Stud. doi:10.1111/joss.12225.

7. Martin A. Revolt over Cadbury's 'rounder, sweeter' bars: Not only has the classic rectangle shape of a Dairy Milk changed, customers say they are more 'sugary' too. Daily Mail. 2013;29:528-41. Downloaded from http:// www.dailymail.co.uk/news/article-2421568/Revolt-Cadburys-roundersweeter-bars-Not-classic-rectangle-shape-Dairy-Milk-changed-customersalso-sugary.html.

8. Ngo M, Misra R, Spence C. Assessing the shapes and speech sounds that people associate with chocolate samples varying in cocoa content. Food Qual Prefer. 2011;22:567-72.

9. Ngo MK, Spence C. Assessing the shapes and speech sounds that people associate with different kinds of chocolate. J Sens Stud. 2011;26:421-8.

10. Bremner A, Caparos S, Davidoff J, de Fockert J, Linnell K, Spence C. Bouba and Kiki in Namibia? A remote culture make similar shape-sound matches, but different shape-taste matches to Westerners. Cognition. 2013;126:165-72.

11. Fairhurst M, Pritchard D, Ospina D, Deroy O. Bouba-Kiki in the plate: combining crossmodal correspondences to change flavour experience. Flavour. 2015;4:22.

12. Stewart PC, Goss E. Plate shape and colour interact to influence taste and quality judgments. Flavour. 2013;2:27.

13. Spence C, Piqueras-Fiszman B. The perfect meal: the multisensory science of food and dining. Oxford: John Wiley \& Sons; 2014.

14. Piqueras-Fiszman B, Alcaide J, Roura E, Spence C. Is it the plate or is it the food? Assessing the influence of the color (black or white) and shape of the plate on the perception of the food placed on it. Food Qual Prefer. 2012;24:205-8.

15. Velasco C, Salgado-Montejo A, Marmolejo-Ramos F, Spence C. Predictive packaging design: tasting shapes, typefaces, names, and sounds. Food Qual Prefer. 2013;34:88-95.

16. Velasco C, Woods AT, Petit O, Cheok AD, Spence C. Crossmodal correspondences between taste and shape, and their implications for product packaging: a review. Food Qual Prefer. 2016;52:17-26.

17. Westerman SJ, Gardner PH, Sutherland EJ, White T, Jordan K, Watts D, Wells S. Product design: preference for rounded versus angular design elements. Psychol Mark. 2012;29:595-605.

18. Lunardo, R., \& Livat, F. (in press). Congruency between color and shape of the front labels of wine: effects on fluency and aroma and quality perceptions. Int J Entrepreneurship Small Bus.

19. Spence C. Managing sensory expectations concerning products and brands: capitalizing on the potential of sound and shape symbolism. J Consum Psychol. 2012;22:37-54
20. Bar M, Neta M. Humans prefer curved visual objects. Psychol Sci. 2006;17: 645-8.

21. Wang Y, Zhang Q. The affective effect of simple geometric shapes. Adv Psychol. 2015;5:471-80.

22. Wang Y, Zhang Q. Affective priming by simple geometric shapes: evidence from event-related brain potentials. Front Psychol. 2016;7:917. doi:10.3389/ fpsyg.2016.00917.

23. Deliza R, MacFie HJH. The generation of sensory expectation by external cues and its effect on sensory perception and hedonic ratings: A review. J Sens Stud. 1996;11:103-28.

24. Piqueras-Fiszman B, Spence C. Sensory expectations based on productextrinsic food cues: an interdisciplinary review of the empirical evidence and theoretical accounts. Food Qual Prefer. 2015:40:165-79.

25. Yeomans MR, Tepper BJ, Rietzschel J, Prescott J. Human hedonic responses to sweetness: Role of taste genetics and anatomy. Physiol Behav. 2007:91:264-73.

26. Hovland $\mathrm{Cl}$, Harvey OJ, Sherif M. Assimilation and contrast effects in reactions to communication and attitude change. J Abnorm Soc Psychol. 1957:55:244-52.

27. Schifferstein HNJ. Effects of product beliefs on product perception and liking. In: Frewer L, Risvik E, Schifferstein H, editors. Food, people and society: A European perspective of consumers' food choices. Berlin: Springer Verlag; 2001. p. 73-96.

28. Cardello AV, Sawyer F. Effects of disconfirmed consumer expectations of food acceptability. J Sens Stud. 1992;7:253-77.

29. Carlsmith JM, Aronson E. Some hedonic consequences of the confirmation and disconfirmation of expectances. J Abnorm Soc Psychol. 1963;66:151-6.

30. Shermer DZ, Levitan CA. Red hot: the crossmodal effect of color intensity on perceived piquancy. Multisensory Res. 2014;27:207-23.

31. Woods AT, Lloyd DM, Kuenzel J, Poliakoff E, Dijksterhuis GB, Thomas A. Expected taste intensity affects response to sweet drinks in primary taste cortex. Chem Senses. 2011;22:365-9.

32. Cohen J. Statistical power analysis for the behavioral sciences. 2nd ed. Hillsdale: Lawrence Erlbaum Associates; 1988.

33. Bartoshuk LM, Fast K, Snyder DJ. Difference in our sensory worlds: invalid comparisons with labelled scales. Curr Dir Psychol Sci. 2005;14:122-5.

34. O'Mahony M, Goldenberg M, Stedmon J, Alford J. Confusion in the use of the taste adjectives 'sour' and 'bitter'. Chem Senses Flavour. 1979:4:301-18.

35. Salgado-Montejo A, Alvarado JA, Velasco C, Salgado CJ, Hasse K, Spence C. The sweetest thing: the influence of angularity, symmetry, and the number of elements on shape-valence and shape-taste matches. Front Psychol. 2015;6:1382

36. Poulter, S. (2016). Now Cadbury ditches twist-off wrappers on Roses: brand ends almost 80 years of tradition while also changing the shape of two of the chocolates. Daily Mail. Downloaded from http://www.dailymail.co.uk/ news/article-3550961/Now-Cadbury-ditches-twist-wrappers-Roses-Brandends-80-years-tradition-changing-shape-two-chocolates.html.

37. Lenfant F, Hartmann C, Watzke B, Breton O, Loret C, Martin N. Impact of the shape on sensory properties of individual dark chocolate pieces. LWT- Food Sci Technol. 2013;51:545-52.

38. Spence $C$. Assessing the influence of shape and sound symbolism on the consumer's response to chocolate. New Food. 2014;17(2):59-62.

39. Debaste F, Kegelaers $Y$, Liegeois S, Ben Amor H, Halloin V. Contribution to the modeling of chocolate tempering process. J Food Eng. 2008;88:568-75.

\section{Submit your next manuscript to BioMed Central and we will help you at every step:}

- We accept pre-submission inquiries

- Our selector tool helps you to find the most relevant journal

- We provide round the clock customer support

- Convenient online submission

- Thorough peer review

- Inclusion in PubMed and all major indexing services

- Maximum visibility for your research

Submit your manuscript at www.biomedcentral.com/submit 